\title{
A MATHEMATICAL MODELLING APPROACH FOR MULTIOBJECTIVE MULTI-STAGE HYBRID FLOW SHOP \\ SCHEDULING PROBLEM
}

\begin{abstract}
The scheduling of flow shops with multiple parallel machines per stage, usually referred to as the hybrid flow shop (HFS), is a complex combinatorial problem encountered in many real world applications. The problem is to determine the allocation of jobs to the paralel machines as well as the sequence of the jobs assigned to each machine. To solve the problem, a 0-1 mixed integer mathematical model with multiobjective is formulated in order to find out the best solution of the problem. Model outcomes are compared for different weights.
\end{abstract}

Keywords: Hybrid Flow Shop, Mathematical Model, Multicriteria Decision Making Methods

\section{Introduction}

In the classical flow shop problem, a set of jobs flow through multiple stages in the same machine order, where each stage consists of only one machine. A hybrid flowshop scheduling problem (HFSP) consists of a series of production stages, each of which has several machines operating in parallel. Some stages may have only one facility, but for the plant to be qualified as a hybrid flowshop, at least one stage must have several facilities. The important characteristic of hybrid flowshop is that the jobs do not need to be processed at all stages; it means that in HFSP jobs can skip from some workstations.

Recently, the consideration of sequence-dependent setup times (SDST) becomes popular among researchers intending to investigate the scheduling decisions in real manner. In many real-life situations such as chemical, printing, pharmaceutical, and automobile manufacturing (Zandieh, Fatemi Ghomi, \& Moattar Hosseini, 2006), the setup operations, such as cleaning up or changing tools, are not only often required between jobs but they are also strongly dependent on the immediately preceding job on the same machine (Naderi et al., in press). Scheduling problems with SDSTs are among the most difficult classes of scheduling problems. A one-machine scheduling problem with SDST is NP- 
hard (Zandieh et al.,2006). Even for a small system, the complexity of this problem is beyond the reach of existing theories. Therefore, HFFS with SDST as a complex variant of scheduling problems is considered as an NP-hard problem in strong sense (Naderi et al., in press). (F. Jabbarizadeh a, M. Zandieh b, D. Talebi, 2009). In this paper, we intend to schedule k-stage HFS with SDST under different objectives. As described in Fig. 1, there are identical paralel machines at stages. At any time every job can be processed by at most one machine and every machine can process at most one job. We assume that jobs can wait between stages and preemption is not allowed. This problem will be denoted as $\mathrm{HF}_{\mathrm{k}}$, $(\mathrm{PM}(\mathrm{k})) / \mathrm{s}_{\mathrm{jik}} / \mathrm{C}_{\max }$. A mathematical model developed and solved for a multiobjective case.

\section{Literature Review}

During the last year, several interesting literature reviews and surveys have analysed and classified various proposals regarding the HFS problem. The HFS problem is commented in the Works of Vignier, Billaut, and Proust $(1995,1999)$. In Vignier et al. (1999) the work is split into two parts. The first part is focused on two-stage flowshop problems, and the second stage on the general k-stage problem. The authors do not identify any solved problems related to makespan and average flow time Kis and Pesch (2005) and provide an extended literature review about exact methods in HFS. The authors focus on branch and bound $(\mathrm{B} \& \mathrm{~B})$ and constraint propagation techniques. An interesting classification of resolution methods is offered in Quadt and Kuhn (2007). Ribas et al. (2010) recently classified papers according to HFS characteristics and production limitations. This represents a new approach to the classification of papers in the HFS environment.

Heuristic and metaheuristic solution approaches are dominant, but some exact methods are often used for simple cases. Branch and bound (B\&B) and dynamic programming techniques are the main actors on the stage of the exact techniques. In Gupta and Tunc (1991), Brah and Hunsucker (1991), Rajendran and Chaudhuri (1992) or Lee, Cheng, and Lin (1993) various B\&B solutions for minimising makespan are defined. Some authors develop dynamic programming algorithms to solve the problem optimally with makespan criterion. A two-machine flowshop scheduling problem where machines are not always available is studied in Lee (1997) and extended in Lee (1999). A two-stage HFS problem with one machine in the first stage, and two different machines in parallel in the second stage, is discussed in Riane, Artiba, and Elmaghraby (2002). (Pedro Gómez-Gasquet a, $\Uparrow$, Carlos Andrés b, Francisco-Cruz Lario, 2011)

When problems grow in complexity or data volume, authors usually propose approximate methods. For a basic review of HFS under the makespan criterion, several useful papers can be found. Early papers by Shen and Chen (1972) and Sriskandarajah and Sethi (1989) present two heuristics based on the Johnson algorithm; while Gupta (1988) introduces a new heuristic based on the longest processing time index. 
Extensive works has been done in hybrid flowshop scheduling. Most studies assume that either no setup has to be performed or that setup times are sequenceindependent (Allahverdi, Ng, Cheng, \& Kovalyov, 2008). Botta-Genoulaz (2000) proposed several heuristics for a flowshop with multiple identical machines per stage, positive time lags and out-tree precedence constraints as well as sequenceindependent setup and removal times. Harjunkoski and Grossmann (2002) included setup times in their work but are only dependent on the machine and not on the job. Kurz and Askin (2003) compared several methods for a makespan minimization problem with sequence-dependent setup times. Jobs are allowed to skip stages. They also developed an integer model and some heuristics and a random keys genetic algorithm for SDST flexible flowshop (Kurz \& Askin, 2004). Andres (2005) considered the problem of products grouping in a tile industry. They proposed some heuristic and metaheuristic methods for a threestage HFSP with sequence-dependent setup times. For SDST hybrid flowshop an immune algorithm (IA) is proposed by Zandieh, Fatemi Ghomi, and Moattar Husseini (2006). Gholami, Zandieh, and Alem-Tabriz (2008) showed how they can incorporate simulation into genetic algorithm approach to the scheduling of a sequence-dependent setup time hybrid flowshop with machines that suffer stochastic breakdown to optimize objectives based on expected makespan. Moreover, Naderi, Zandieh, Khaleghi Ghoshe Balagh, and Roshanaei (2009) hybridized the simulated annealing with a simple local search to solve the hybrid flowshops scheduling with sequence-dependent setup times, transportation times and total completion time and total tardiness as objective functions. (J. Behnamian, M. Zandieh / Expert Systems with Applications 38 (2011))

\section{Hypotheses/Objectives}

Machine scheduling problems usually have different objectives and the job order strongly depends on its objectives. A mixed integer programming model is presented to show this dependency and the change in terms of different objective weighs.

\section{Research Design/Methodology}

We intend to schedule k-stage HFS with SDST under minimization of makespan. As described in Fig. 1, there are identical paralel machines at stages. At any time every job can be processed by at most one machine and every machine can process at most one job. We assume that jobs can wait between stages and preemption is not allowed. This problem will be denoted as $\mathrm{HF}_{\mathrm{k}},(\mathrm{PM}(\mathrm{k})) / \mathrm{s}_{\mathrm{ijk}} /$ different objectives. 


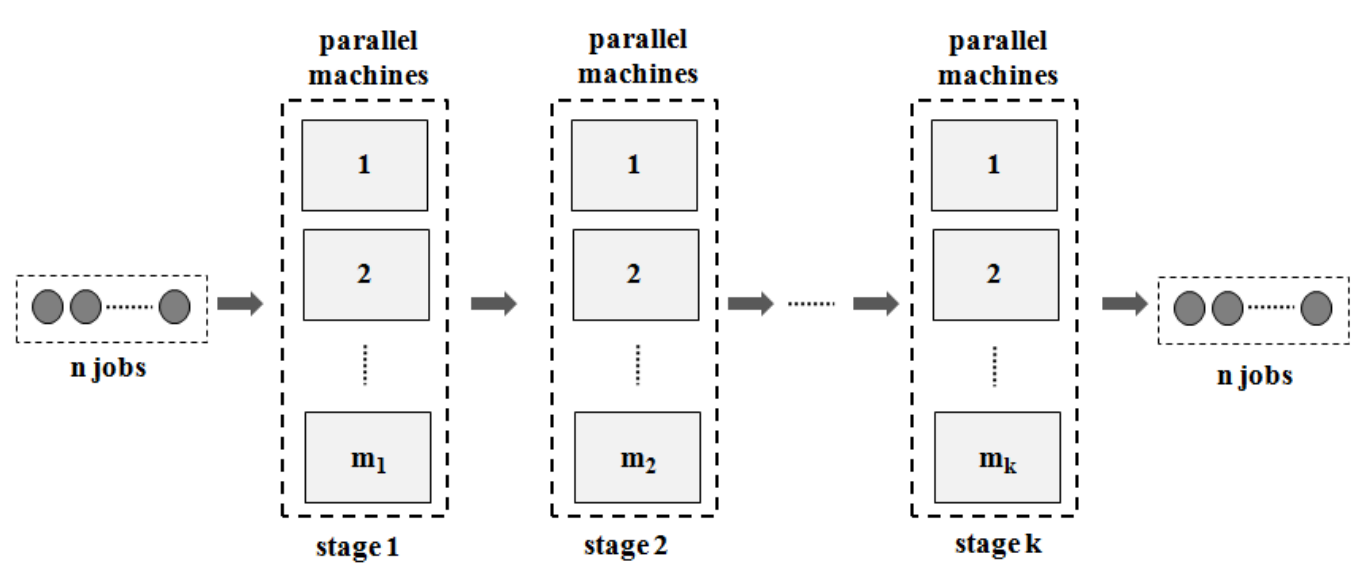

\section{Data/Model Analysis}

We provide a $0-1$ mixed integer linear programming formulation for the problem under consideration.

Indexes:

$\mathrm{i}, \mathrm{j} \in \mathrm{N} \quad$ jobs

$\mathrm{s} \in \mathrm{N} \quad$ job sequence

$1 \in \mathrm{M} \quad$ machines

$\mathrm{t} \in \mathrm{K} \quad$ stages

Sets:

$\mathrm{N}=\{1,2, \ldots, \mathrm{n}\}$ job

$\mathrm{M}=\left\{1,2, \ldots, \mathrm{m}_{\mathrm{t}}\right\} \quad$ machine

$\mathrm{K}=\{1,2, \ldots, \mathrm{k}\}$ stage

\section{Parameters:}

n : number of jobs

$\mathrm{k} \quad$ : number of stages

$\mathrm{m}_{\mathrm{t}} \quad$ : number of machines at stage $\mathrm{t}$

$\mathrm{p}_{\mathrm{jt}} \quad$ : processing time for job $\mathrm{j}$ at stage $\mathrm{t}(\mathrm{sn})$

$\mathrm{h}_{\mathrm{jt}} \quad$ : setup time of job $\mathrm{j}$ if job $\mathrm{j}$ is assigned to at the first position at stage $\mathrm{t}(\mathrm{sn})$

$\mathrm{s}_{\mathrm{jij}} \quad$ : sequence-dependent setup time from job $\mathrm{i}$ to job $\mathrm{j}$ at stage $\mathrm{k}(\mathrm{sn})$

$\mathrm{q}_{\mathrm{j}} \quad$ : demand for job $\mathrm{j}$

$\mathrm{M} \quad$ : big reel number

Decision Variables:

$\mathrm{C}_{\mathrm{jt}} \quad$ : completion time of $\mathrm{job} \mathrm{j}$ at stage $\mathrm{t}$

$\mathrm{C}_{\max }:$ makespan 
ISAHP Article: A Style Guide for Paper Proposals To Be Submitted to the International Symposium on the Analytic Hierarchy Process 2016, London, U.K.

$$
\mathrm{X}_{\mathrm{jtls}}=\begin{gathered}
\dot{i} \\
1, \wedge \text { if job jis allocated s of machine lat staget } \\
\dot{i} \\
{[0, \wedge \text { otherwise } i}
\end{gathered}
$$

Mathematical formulation

The problem can be formulated as follows:

Minimize $\mathrm{C}_{\max }$ (may change according to different objectives)

subject to:

$$
\begin{aligned}
& \sum_{l=1}^{m_{t}} \sum_{s=1}^{n} X_{j t l s}=1 \quad \forall(j, t)(j, s) \in N, l \in M, t \in K \\
& \sum_{j=1}^{n} X_{j t s} \leq 1 \\
& \forall(t, l, s)(j, s) \in N, l \in M, t \in K \\
& C_{j t}+M *\left(1-X_{j t s}\right) \geq C_{j|t-1|}+p_{j t} * q_{j}+h_{j t} \\
& \forall(j, l) s=1, t>1,(j, s) \in N, l \in M, t \in K \\
& C_{j t}-C_{i t}+M *\left(2-X_{i t l(s-1)}-X_{j t l s}\right) \geq p_{j t} * q_{j}+s_{i j t} \\
& \forall(i, j, t, l) i \neq j, s>1,(i, j, s) \in N, l \in M, t \in K \\
& C_{j t} \geq C_{j(t-1)}+p_{j t} * q_{j} \quad \forall(j) \quad t>1, j \in N, t \in K \\
& \sum_{j=1}^{n} X_{j t s}-\sum_{i=1}^{n} X_{i t l(s-1)} \leq 0 \\
& \forall(t, l) s>1, i \neq j,(i, j, s) \in N, l \in M, t \in K \\
& C_{\max } \geq C_{j t} \quad \forall(j, t) \quad j \in N, t \in K \\
& C_{j t} \geq 0 \\
& \forall(j, t) \quad j \in N, t \in K \\
& X_{j t s} \in\{0,1\} \quad \forall(j, t, l, s)(j, s) \in N, l \in M, t \in K
\end{aligned}
$$

\section{Limitations}

Since different constraints and assumptions can result in different scheduling problems in hybrid flowshops, we introduce the following characters which are considered in this paper. Besides since the problem has NP-hard structure, for big sized problems, it is difficult to find an optimal solution by using the mathematical model.

1. All data in all problems used are known deterministically when scheduling is undertaken.

2. Number of jobs, machines and stages are fixed. Each stage has at least one machine, and at least one stage must have more than one machine. 
3. A machine can process only one job at a time.

4. Each job must be processed by at most one machine in each stage.

5. A job once started on the machine must be completed on it without interruption.

6. Jobs are available for processing at a stage immediately after releasing from the previous stage.

7. Transportation times between the stages are negligible.

8. Machines are available with no breakdowns.

In the above formulation, the objective is to determine a schedule that minimizes a makespan. Different objectives are weighted by using an AHP model and the model outcomes are compared.

Constraint set (1) ensures that job $\mathrm{j}$ at stage $\mathrm{t}$ is assigned to only one sequence position on a machine. Constraint sets (2) guarantees that a machine can process at most one job at a time.

Constraint (3) specifies the completion time of job j, if the job is assigned to the first sequence on machine 1 at stage $t$, is greater than the total of processing time of job $\mathrm{j}$ and the setup time of job $\mathrm{j}$. The value of $\mathrm{M}$ is set to a very large constant.

Constraint (4) determines the completion time of job $\mathrm{j}$, (if job $\mathrm{j}$ is assigned to second or greater sequence on machine 1 at each $t$ and if job $j$ is immediately scheduled after job $i$ ), is greater than or equal to the total of the processing time of job $\mathrm{j}$ and the sequence dependent setup time from $\mathrm{i}$ to $\mathrm{j}$.

Constraint (5) specifies the conjunctive precedence constraints for the jobs, which says a job can not start processing at stage $t$ before it is processed at stage $t-1$. Constraint (6) ensures the assignment of jobs successively. Constraint (7) determines the makespan that is grater than all completion times. Constraint (8) and Constraint (9) represent the state of the decision variables.

It is noted that an optimal solution can be obtained by running a commercial mathematical programming software, GAMS 24.2.2 with IBM ILOG CPLEX optimizer and Intel ${ }^{\circledR}$ Core $^{\mathrm{TM}}$ i5 CPU / $2.50 \mathrm{GHz} / 4$ GB RAM. We have found that the mathematical model can be used for solving problems with up to "2 stages, 3 machines, 8 jobs", " 4 stages, 5 machines, 7 jobs", "6 stages, 7 machines, 6 jobs" in acceptable time.

\section{Conclusions}

We considered a k-stage hybrid flow shop scheduling problem with sequence dependent setup times for minimizing the makespan and for some other different objectives. A 0-1 mixed integer mathematical model is formulated in order to find the best solution of the problem. Since the job order strongly depends on the 
ISAHP Article: A Style Guide for Paper Proposals To Be Submitted to the International Symposium on the Analytic Hierarchy Process 2016, London, U.K.

problem objective, we define different objectives and weight them in order to see their effect on the final job schedule. An ANP model is used and the weights are incorporated to the mathematical model as objective coefficients.

\section{Key References}

Zandieh, M., Fatemi Ghomi, S.M.T., Moattar Husseini, S.M., (2006): An immune algorithm approach to hybrid flow shops scheduling with sequencedependent setup times, Applied Mathematics and Computation 180 (1), 111-127.

Behnamian, J., Zandieh, M., (2011): A discrete colonial competitive algorithm for hybrid flowshop scheduling to minimize earliness and quadratic tardiness penalties, Expert Systems with Applications 38, 1490-1498.

Gómez-Gasquet, P., Andrés, C., Lario, F.C., (2012): An agent-based genetic algorithm for hybrid flowshops with sequence dependent setup times to minimise makespan, Expert Systems with Applications 39, 8095-8107.

Gupta, J.N.D., 1988: Two-stage, hybrid flow shop scheduling problem, Journal of the Operational Research Society 39 (4), 359-364.

Jabbarizadeh, F., Zandieh, M., Talebi, D., 2009: Hybrid flexible flowshops with sequence-dependent setup times and machine availability constraints, Computers \& Industrial Engineering 57, 949-957.

Lee, C.Y., Vairaktarakis, G.L., (1994): Minimizing makespan in hybrid flowshops, Operations Research Letters 16 (3) 149-158.

Ribas, I., Leisten, R., \& Framiñan, J. M., (2010): Review and classification of hybrid flow shop scheduling problems from a production system and a solutions procedure perspective, Computers \& Operations Research 37, 1439-1454.

Ruiz, R., Vazquez-Rodriguez, J.A., 2010: The hybrid flow shop scheduling problem, European Journal of Operational Research 205, 1-18.

Pinedo, M., (2002): Scheduling: Theory Algorithms and Systems, second ed., Prentice Hall, Upper Saddle, NJ.

Zandieh, M., Fatemi Ghomi, S.M.T., Moattar Husseini, S.M., (2006): An immune algorithm approach to hybrid flow shops scheduling with sequencedependent setup times, Applied Mathematics and Computation 180 (1), 111-127. 